\title{
The P1 Plasmid-Partition System Synthesizes Two Essential Proteins from an Autoregulated Operon'
}

\author{
Stanley A. Friedman and Stuart J. Austin \\ Laboratory of Chromosome Biology, BRI-Basic Research Program, NCI-Frederick Cancer Research Facility. \\ Frederick, Maryland 21701
}

Received December 18, 1987

\begin{abstract}
The P1 partition region contains two large open reading frames that encode the proteins ParA and ParB. It was previously shown that ParA is essential for partition activity. Using a novel assay, we show that ParB protein is also an absolute requirement for partition and that it is active in trans to the partitioning plasmid. Development of complementation tests for par $A$ and par $B$ allowed us to assign a number of partition-defective mint mutants of a $P 1$ miniplasmid to the par $A$ and par $B$ cistrons. Using gene fusion techniques, it was shown that par $A$ and par $B$ constitute an operon controlled from a promoter proximal to the start of parA. Transcription from this promoter is autoregulated by a feedback loop that is sensitive to the ParA and ParB proteins in concert. The parB gene also appears to be expressed at a low level from a second promoter at the intercistronic boundary. This results in a low level of expression and tight autoregulation for the ParA protein and slightly less stringent control for ParB synthesis.
\end{abstract}

Plasmids such as the sex factor $F$ and plasmid prophage of bacteriophage $P 1$ are stably maintained in their Escherichia coli host despite having a copy number of approximately 1 per host chromosome (Prentki et al., 1977; Collins and Pritchard, 1973). In addition to strictly regulated replication systems, these plasmids encode precise partition mechanisms to ensure the proper distribution of plasmid copies to daughter cells (Ogura and Hiraga, 1983; Austin and Abeles, 1983a).

The plasmid region responsible for partition of P1 has been isolated and the DNA sequence determined (Abeles et al., 1985). Analysis of the sequence showed that the region consisted of two large open reading frames, par $A$ and parB, that could encode proteins of 42 and $39 \mathrm{kDa}$, respectively. Downstream from the open reading frames is a region of about $100 \mathrm{bp}$ that is important in determining plasmid incompatibility

\footnotetext{
1 The U.S. Government's right to retain a nonexclusive royalty-free license in and to the copyright covering this paper, for governmental purposes, is acknowledged.
}

(incB). The incB region also contains a cisacting recognition site (par $S$ ) that is essential for partition (Martin et al., 1987). It is proposed that this site specifies the attachment of the daughter plasmids to some host cell structure responsible for the selective movement of the copies to daughter cells during cell division (Abeles et al., 1985).

The two open reading frames both produce proteins, designated ParA and ParB, respectively (Abeles et al., 1985). A nonsense (amber) mutation parA17 in the parA open reading frame was shown to cause a suppressible partition-defective phenotype in a P1 miniplasmid. This mutation was complemented in trans by a product of the wild-type parA, parB, region (Abeles et al., 1985). This argued that the ParA protein is essential for partition, although alternative interpretations were possible. It was also shown that overproduction of the ParA protein from a multicopy plasmid carrying an isolated par $A$ cistron blocked partition of a resident $\mathbf{P} 1$ miniplasmid. This suggested that controlled synthesis of ParA protein is important for function. Here we show that both ParA and 
ParB are essential trans-acting requirements for partition and show that they are subject to an autoregulatory control system that results in controlled production.

\section{MATERIALS AND METHODS}

Bacteria and phage strains. Bacterial strains used were N100 recA13, galK, pro, $S^{R}$ (Das et al., 1976), CM5649 polA, sup $D^{\text {ts }}$, tet $^{\mathrm{r}}$, $\operatorname{trp}$ (am), (Kelley, 1980), and SE4006 (constructed by Scott Emr; araD, $\Delta$ lac169, relA, thi, rpsL, recA56 srl:Tn10). Phage $\lambda-\mathrm{P} 1: 5 \mathrm{R}$ and the isolation of putative par point mutants of it are described elsewhere (Austin and Abeles, 1983b). The imm $\lambda$ clear plaque selector phages used to select $\lambda$ lysogens were previously described (Austin et al., 1982).
Media, buffers, and standard manipulations. Media, buffers, and standard manipulations of cells, phages, and bacterial strains were as previously described (Abeles et al., 1984). $\beta$-Galactosidase assays in $E$. coli SE4006 background and the use of $X$-gal plates as an indicator for $\beta$-galactosidase activity were performed as previously described (Miller, 1972).

Plasmid constructions. The map positions of P1 inserts in the plasmids used in these studies are shown in Fig. 1. The plasmid pALA823 was constructed by inserting the BamHI-PstI fragment of pALA261 (Abeles et al., 1985) into the BamHI-PstI sites of the pSC101-based vector pGB2 (Churchward et al., 1984). The $\mathrm{N}$-terminal portion of the par $B$ open reading frame was deleted from pALA 823 by replacing the BglII-PstI frag-
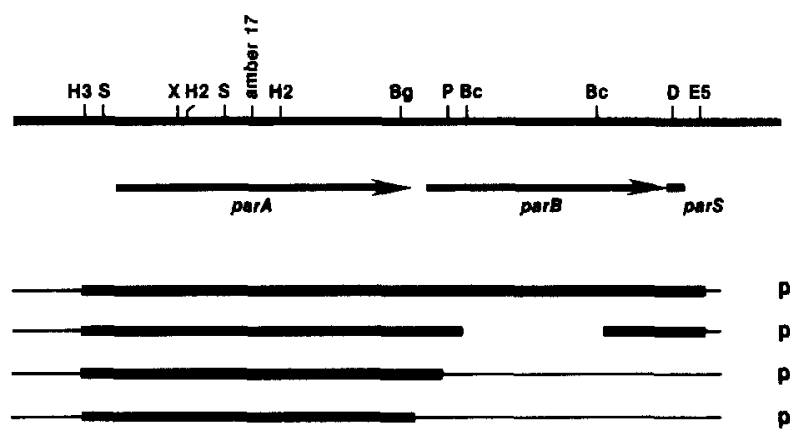

PALABO 1

PALA803

PALA823

PALA824

PALABO7

PALA826

PALA809

PALAB17

[Fuston Type]

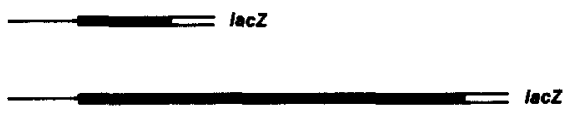

[Protain] pALA175

[Proteln] pALABO4

[Operon] PALA811

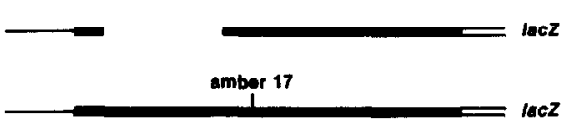

[Operon]

pALAB15

[Protein] pALAB10

[Operon] pALAB12

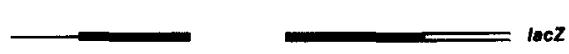

[Operon] pALA829

FIG. 1. Plasmids used in this study. The restriction sites of the P1 par region are as follows: H3, HindIII; S, SphI; X, Xhol; H2, HincII; Bg, BglII; P, PvuI; Bc, BclI; D, DraI; E5, EcoRV. The blunt-end cutters DraI and EcoR 5 were converted to BamHI as described (Abeles et al., 1985). 
ment with a double-stranded synthetic oligonucleotide whose projecting ends were complementary to the $B g l \mathrm{II}$ and PstI sites and had the sequence GATCTAACTGATAACTGCA where the complementary strand had the 4-base overhang for the PstI site. This insert reconstructs the carboxy terminus of the parA open reading frame and its stop signal resulting in plasmid pALA824. The plasmid pALA807 was constructed by inserting the XhoI-BamHI fragment from pALA271 (Abeles et al., 1985) between the SalI and BamHI sites of pGB2. A plasmid which expresses parB from the $\operatorname{trp}$ promoter (pALA826) was constructed by inserting a 90-bp fragment containing the trp promoter (Pharmacia, Inc.) into the HindIII-BglII site of pALA807. The plasmid pALA809 was constructed by inserting the HindIII-BamHI fragment of pALA271 into the HindIIIBamHI sites of pGB2. The plasmid pALA817 was constructed by a deletion of the parA gene of pALA 809 generated by $H$ incII digestion and religation.

Plasmid pFAMK (Koop et al., 1987) is a derivative of colE1 which contains the rep region of the $\mathrm{F}$ plasmid as the 3.3-kb KpnI fragment ( $F$ 43.6-46.9). This includes an active replication region but only a small inactive portion of the F partition region (Ogura and Hiraga, 1983). Plasmid pALA607 was constructed by removing the $1.4-\mathrm{kb}$ PstI fragment of pFAMK, thus deleting part of the bla gene that confers ampicillin resistance.

The protein fusion plasmids were constructed as follows: The plasmid pALA175 was constructed by inserting a HindIII-XhoI fragment of pALA801 into the HindIII-SalI sites of the protein fusion vector pORF1 (Weinstock et al., 1983). A parB:lacZ protein fusion with an intact parA promoter and gene was constructed by inserting a $E c o$ RI$B c l$ fragment of pALA801 into the EcoRIBamHI sites of pMB1034 (Silhavy et al., 1984) resulting in pALA804. An amber mutation in the parA open reading frame was introduced into pALA 804 by replacing the wild-type $X h o I-B g l$ II fragment with that from pALA104 (Austin and Abeles, 1983b) to form pALA810.

The operon fusion plasmids were constructed as follows: The operon fusion vector pMLB1 109 is a pBR322-based plasmid containing transcription terminators preceding a polylinker cloning site, translation termination signals in all reading frames, and a promoterless $l a c Z$ gene (Berman, unpublished). This was modified by insertion of a HindIII linker (CCAAGCTTGG) into the Smal site resulting in pALA403. The plasmid pALA 811 was constructed by inserting the $H$ indIII-BclI fragment of the P1 par region into the HindIII-BamHI sites of pALA403. A parA17 (amber) version of pALAL811, pALA812, was constructed by replacing the wild-type $X h o I-B g l$ II fragment with that from pALA 104. A parA promoter deletion version, pALA815, was constructed by deletion of a 489-bp $S p h I$ fragment from pALA811. The parA operon fusion plasmid pALA829 was constructed by insertion of the HindIII- $B g l$ II fragment of the parA region from pALA817 into the HindIIIBamHI sites of pALA403.

The "pick-up" assay for partition function. The scheme for this assay is shown in Fig. 2. The assay uses an additional plasmid, the "pick-up vector," that contains a functional unit-copy origin of replication from either PI or F. Homologous recombination between the pick-up vector and the target plasmid results in a composite plasmid containing both the unit-copy origin of replication and the partition region to be tested. The composite plasmid is introduced into an $E$. coli polA ${ }^{-}$ strain in which the high-copy-number replication origins present on the composite cannot function and the F or Pl origin supports replication. Partition activity is measured by scoring for plasmid retention as determined by antibiotic resistance after growth in nonselective medium.

C600 cells carrying pALA136 (P1 origin of replication) or pALA607 (F origin of replication) were transformed with the desired test plasmid containing fragments from the par region using antibiotic selection for the 


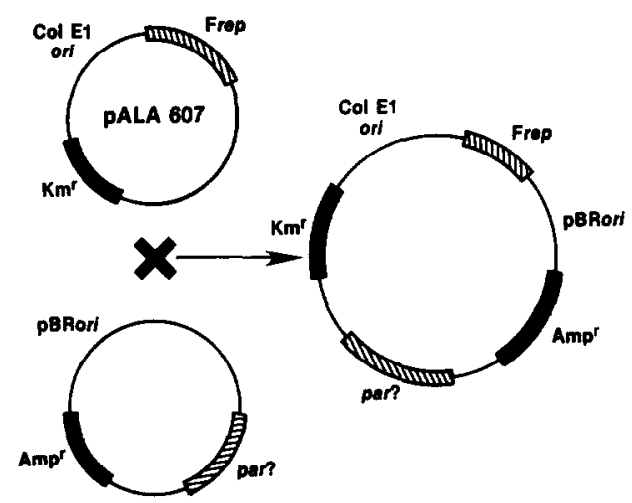

FIG. 2. Scheme for the "pick-up" assay used to determine partition activity of the par regions cloned into the high-copy-number vector pBR322. The partition clone under study recombines with a colE1 derivative containing a unit-copy origin of replication via the extensive homology around the high-copy-number origins of the two plasmids. The composite dimer is transformed into an $E$. coli polA strain which only permits the unit-copy origin of replication to function. Partition activity is measured by determining the rate of loss of the plasmid from a population of cells growing without selection.

maintenance of both plasmids. The total plasmid pool from a 2-ml culture of these cells was isolated by $\mathrm{NaOH}-\mathrm{SDS}$ rapid plasmid preparation procedure as described (Maniatis et al., 1982). Strain CM5649 ( $p o l A^{-}$) was transformed with this mixture by the calcium chloride method (Maniatis $e t$ al., 1982) selected for the antibiotic-resistance determinant of the target plasmid. As the target plasmid containing the selected marker is unable to replicate, only composite plasmids formed by recombination with the pick-up vector can transform. As expected, all transformants subsequently proved to carry both the antibiotic resistance derived from the target plasmid and that derived from the pick-up vector.

Each transformation mixture consisted of the entire yield of plasmid DNA added to calcium treated cells from a 3-ml culture. Typically, this yielded a total of approximately 50 antibiotic-resistant colonies. Colonies were restreaked once on the same medium and the resulting colonies picked to $\mathrm{L}$ broth with antibiotic for overnight growth. Overnight cultures were diluted into fresh medium with antibiotic at $5 \times 10^{6} \mathrm{cells} / \mathrm{ml}$. When the cell density reached $1 \times 10^{8}$ cells/ $\mathrm{ml}$, the cells were diluted 1:1000 for 10 generations of unselected growth, or 1:10 $0^{6}$ for 25 generations of growth, in L broth without antibiotics at $37^{\circ} \mathrm{C}$. Samples of cells were taken before and after the growth period without antibiotic selection. Individual colonies derived by plating these samples on antibiotic No. 2 agar were picked to antibiotic medium No. 2 with and without antibiotic selection to score for the retention of composite plasmid. In those cases where a loss event was recorded, both antibiotic resistances were lost concomitantly (in $>95 \%$ of cases), proving that the component plasmids were indeed associated in a composite.

Stability tests for $\lambda-P 1: 5 R$ derivatives. Lysogens containing $\lambda-P 1: 5 R$ and its derivatives were constructed as previously described (Austin et al., 1982). The loss of the plasmid prophage during 25 generations of unselected growth was measured as previously described (Austin et al., 1982).

\section{RESULTS}

In order to characterize the partition region of $\mathrm{P} 1$, various fragments of the region have been cloned into the high-copy vector pBR322. The use of high-copy-number vectors greatly facilitates the manipulation of such sequences but normally prevents the direct assay of partition activity, as high-copy vectors do not require a precision partition mechanism for stable maintenance. Previously, we developed a direct assay for partition function, using a strain that limits pBR322 replication to give a relatively low copy number. Materials and Methods in Fig. 2 describe a new method, the "pick-up" assay, that combines the advantages of a unit-copy replication system with the convenience of direct use of pBR322 clones. The method consists of the selection of plasmid composites formed by recombination between the pBR322-based plasmid to be tested and a "pick-up vector" consisting of a second, differentially marked, pBR322 de- 
rivative carrying an $\mathrm{F}$ or $\mathrm{P} 1$ replication region.

The minimal P1 partition region present in pALA801 supported the partition of composites driven by the $F$ origin of replication (Table 1). Similar results were obtained using a plasmid containing the $P 1$ origin of replication instead of the F origin (data not shown). As with our previous observation using P1 miniplasmids (Austin and Abeles, 1983b; Austin et al., 1982; Abeles et al., 1985), an amber mutation in the parA open reading frame or a deletion that removes the incB sequence from the par region rendered the composite highly unstable (data not shown), suggesting that the ParA protein and the incB sequence are both essential for par function.

\section{The ParB Protein Is an Essential trans- Acting Requirement for Partition}

The par $B$ open reading frame lies between par $A$ and the inc $B$ sequence and produces a protein product, $\mathrm{ParB}$, with an apparent molecular mass on SDS-PAGE of $44 \mathrm{kDa}$ (Abeles et al., 1985).

Plasmid pALA803 was derived from pALA801 by deletion of the 498-bp BclI fragment within parB and results in an abbreviated ParB protein with 166 amino acids missing from its central region (Abeles et al., 1985). A comparison of pALA803 with its pALA801 parent in the pick-up assay showed that pALA803 has no apparent partition activity (Table 1), suggesting that an intact parB open reading frame is essential for par function.

Plasmid pALA807 which contains an isolated $\operatorname{par} B$ open reading frame, was constructed (Fig. 1). It is based on the pSC101derived vector $\mathrm{pGB} 2$ and can therefore be stably maintained in the polA cells used in the partition assay. Versions of strain CM5649 polA $A^{-}$that contain pALA807 or its pGB2 parent were constructed. Pick-up assays carried out using these strains showed that the partition defect caused by the parB deletion in pALA803 is suppressed when the par $B$ open reading frame is present in trans (Table 1). We conclude that the ParB protein is essential for partition and is trans-acting. ParB could also be provided from a pGB2based plasmid that carries both the parA and the parB open reading frames (pALA809). This plasmid could also suppress the parA mutation parA17, showing that both ParA and $\mathrm{ParB}$ are active in trans from this construct (Table 1). It can be concluded that both the ParA and the ParB proteins play a role in partition. As the phenotypes imposed by the par $A$ and $\operatorname{par} B$ lesions are equally severe and indistinguishable from those of constructs that have no partition region at all, it can further be concluded that both proteins are essential for any active partition to occur.

\section{Assignment of Existing P1 par Mutants to the parA and parB Cistrons}

We have previously described the isolation of a collection of mutants of the P1 mini-

TABLE 1

Percentage of Plasmids Retained after 25 Generations of Unselected Growth Using a Plasmid CONTAINING THE F ORIGIN OF REPLICATION (pALA607) TO "PICK UP" AND MAINTAIN THE Plasmid UNDER STUdY at LOW COPY NUMBER

\begin{tabular}{llccc}
\hline & & \multicolumn{2}{c}{$\begin{array}{c}\text { Percentage plasmid retention; } \\
\text { host strain CM5649 containing: }\end{array}$} \\
\cline { 3 - 4 } Plasmid & Construct & $\begin{array}{c}\text { pGB2 } \\
\text { (control) }\end{array}$ & $\begin{array}{c}\text { pALA807 } \\
(\text { parB })\end{array}$ & $\begin{array}{c}\text { pALA809 } \\
(\text { parA, parB })\end{array}$ \\
\hline pALA801 & parA, parB, incB & 97 & 85 & 59 \\
pALA803 & parA, $\Delta$ parB, incB & 2 & 90 & 61 \\
\hline
\end{tabular}


TABLE 2

assignment of PI Partition Mutants TO CISTRONS

\begin{tabular}{|c|c|c|c|}
\hline \multirow[b]{2}{*}{ Plasmid } & \multicolumn{3}{|c|}{ Percentage plasmid retention ${ }^{\alpha}$ in strain } \\
\hline & N100 & $\begin{array}{c}\text { N100 } \\
\text { pALA807 } \\
(\text { par } B)\end{array}$ & $\begin{array}{c}\mathrm{N} 100 \\
\text { pALA809 } \\
(\text { parA, parB) }\end{array}$ \\
\hline$\lambda-\mathrm{P} 1: 5 \mathrm{R}$ & 100 & 100 & 100 \\
\hline$\lambda-P 1: 5 R$ par-8 & $<2$ & 79 & 100 \\
\hline$\lambda-\mathrm{P} 1:$ SparA $17(\mathrm{am})$ & $<2$ & $<2$ & 97 \\
\hline$\lambda-P 1: 5 R$ par-19 & $<2$ & 100 & 74 \\
\hline$\lambda-P 1: 5 R$ par-23 & 28 & 100 & 100 \\
\hline$\lambda-P 1: 5 R$ par-29 & $<2$ & 98 & 98 \\
\hline$\lambda-P 1: 5 R$ par-32 & 23 & 96 & 90 \\
\hline$\lambda-P 1:$ Rpar-34 & $<2$ & $<2$ & 96 \\
\hline$\lambda-P 1: 5 R$ par-35 & $<2$ & $<2$ & 92 \\
\hline$\lambda-P 1: 5 R$ par-36 & $<2$ & 66 & 97 \\
\hline$\lambda-P 1: 5 R$ par-37 & $<2$ & 98 & 94 \\
\hline
\end{tabular}

"Stability tests werc performed by screening for loss of $\lambda$ immunity after 25 generations of unselected growth as described under Materials and Methods. As these mutants can now be assigned to cistrons, we propose to rename them as $\lambda$-PI:SRparB8, $\lambda$-P1:5RparA35, etc.

plasmid $\lambda$-P1:5R that have defects in plasmid maintenance (Austin and Abeles, 1983b). Of 42 mutants, 14 were candidates for having partition defects because they could replicate as plasmids but were lost at a high rate from the growing population. One of these mutants contained the parA17 amber mutation and was located within the parA open reading frame by DNA sequencing (Abeles et al., 1985). Of the remaining 13 mutants, 4 were shown to be leaky mutations affecting the essential replication protein RepA as they were complemented by rep $A$ in trans (Austin et al., 1985). The 10 potential par mutants (including $\lambda-\mathrm{P} 1: 5 \mathrm{R}$ parA17) were tested for complementation by using strains containing pALA807 or pALA809 to supply ParB or ParA and ParB, respectively. The results given in Table 2 show that 7 of the mutants have par $B$ mutations and 3 , including parA17, are parA mutants. There is no consistent difference in the severity of the partition defect between the two mutant classes, although two of the parB mutants appear to be leaky (Table 2).

\section{The Regulation of Par Protein Synthesis}

The regulation of ParA protein synthesis was investigated by use of a parA:lac $Z$ protein fusion construct. In this construct, pALA175, the parA open reading frame is fused at the unique $X h o I$ site to the $l a c Z$ gene such that a fusion protein that consists of the first 54 amino acids of ParA and an active portion of the $\beta$-galactosidase protein is produced (Fig. 1). Despite being expressed from a high-copy-number plasmid, the $\beta$-galactosidase activity of this construct is low (ca. 6 Miller units, Table 3). Moreover, this expression is further attenuated by autoregulation as shown in Table 3. The GB2-based constructs that supply the Par proteins in trans are compatible with the pBR322-based fusion plasmid and can therefore be used to study the effect of the wild-type proteins on expression of the fusion gene. The presence of ParA in trans to the fusion reduced expression threefold. When both ParA and

TABLE 3

par:lacZ PROTEIN FUSION ACTIVITY

\begin{tabular}{lccccc}
\hline & & \multicolumn{3}{c}{ Miller Units in strain containing $^{a}$} \\
\cline { 3 - 6 } $\begin{array}{l}\text { Plasmid } \\
\text { number }\end{array}$ & Construct & $\begin{array}{c}\text { pGB2 } \\
\text { (control) }\end{array}$ & $\begin{array}{c}\text { pALA824 } \\
\text { (ParA) }\end{array}$ & $\begin{array}{c}\text { pALA826 } \\
\text { (ParB) }\end{array}$ & $\begin{array}{c}\text { pALA809 } \\
\text { (ParA, ParB) }\end{array}$ \\
\hline $\begin{array}{l}\text { pALA175 } \\
\text { pALA810 }\end{array}$ & $\begin{array}{c}\text { parA protein fusion } \\
\text { parB protein fusion } \\
\text { parA17(am) }\end{array}$ & $\begin{array}{c}5.9 \\
490\end{array}$ & $\begin{array}{c}2.1 \\
160\end{array}$ & 510 & 0.1 \\
\hline
\end{tabular}

\footnotetext{
${ }^{a} \beta$-Galactosidase assay of par:lacZ protein fusions in $E$. coli SE4006.
} 
ParB were supplied in trans, expression was further reduced to a level indistinguishable from the vector background level (Table 3). The addition of ParB alone did not regulate the expression of parA. Thus, it appears that par $A$ expression can be partially attenuated by its own product but is severely down-regulated when both ParA and ParB are present.

A second protein fusion plasmid that produces a fusion between ParB and $\beta$-galactosidase was constructed (pALA810). This construct contains a full-length parA open reading frame. In order to block the production of ParA from the fusion plasmid which could otherwise affect the outcome of regulation tests, the construct includes a chain-termination mutation early in parA (amber mutation parA17). The results obtained with pALA810 are shown in Table 3. The same basic pattern of regulation was seen when Par proteins were supplied in trans; the expression was shut down 10-fold when ParA and ParB were supplied and a much smaller effect was obtained with ParA alone.

\section{Transcriptional Regulation of lacZ Fusions}

The apparent coordination of regulation of the two Par proteins suggests that the two genes constitute an operon that is transcriptionally regulated from a promoter upstream of parA. If the regulation of par gene expres- sion occurs at the level of transcription, it should affect the expression of a lac $Z$ gene that is under the transcriptional but not the translational control of the region. We therefore constructed a transcriptional fusion in the parA gene at the BglII site, near the end of parA (Fig. 1). In this fusion, the open reading frames are separated so that synthesis of a normal $\beta$-galactosidase protein is dependent on transcription from the parA gene. Using this construct, pALA829, it was found that transcription through the region is remarkably high (giving 18,000 Miller units of $\beta$-galactosidase, Table 4). The addition of ParA in trans to the fusion reduced expression some 3-fold. A further 45-fold reduction was seen when ParA and ParB were both supplied. No effect is seen with ParB alone. Thus, regulation of transcription through parA mimics the regulation of Par protein expression.

We also constructed a transcriptional fusion within the par $B$ gene, using the first $B c l I$ site in $\operatorname{par} B$ as the fusion point. This construct, pALA812, contains 191 bases of the parB open reading frame and has a nonsense mutation, parA17, in the par $A$ gene to block ParA synthesis. Table 4 shows the results obtained with this construct. Transcription through the region gave 5700 Miller units and was subject to a regulatory effect similar to that of the parA region although the re-

TABLE 4

par:lacZ OPERON FUSION ACTIVITY

\begin{tabular}{|c|c|c|c|c|c|}
\hline \multirow{2}{*}{$\begin{array}{l}\text { Fusion } \\
\text { plasmid } \\
\text { present }\end{array}$} & \multirow[b]{2}{*}{ Construct } & \multicolumn{4}{|c|}{ Miller Units in strain containing ${ }^{a}$} \\
\hline & & $\begin{array}{c}\text { pGB2 } \\
\text { (control) }\end{array}$ & $\begin{array}{c}\text { pALA824 } \\
\text { (ParA) }\end{array}$ & $\begin{array}{c}\text { pALA826 } \\
\text { (ParB) }\end{array}$ & $\begin{array}{c}\text { pALA809 } \\
\text { (ParA, ParB) }\end{array}$ \\
\hline pALA829 & parA operon fusion & 18,000 & 6800 & 16,000 & 150 \\
\hline pALA 812 & $\begin{array}{l}\text { parB operon fusion } \\
\text { parA17(am) }\end{array}$ & 5,700 & 2400 & 4,100 & 490 \\
\hline pALA815 & $\begin{array}{l}\text { parB operon fusion } \\
\text { with par } A \\
\text { promoter deleted }\end{array}$ & 180 & 210 & 190 & 170 \\
\hline pALA811 & $\begin{array}{l}\text { parB operon fusion } \\
\quad \text { parA } A^{+}\end{array}$ & 3,600 & 3000 & 510 & 210 \\
\hline
\end{tabular}

\footnotetext{
${ }^{a} \beta$-Galactosidase assay of par:lacZ operon fusions in E. coli SE4006.
} 
pression is somewhat less stringent (Table 4).

Although the above results implied that ParB, when in concert with ParA, has a direct effect on regulation, it was possible that the apparent effect of ParB was due to an artifactual difference between the two plasmids used to supply the proteins in trans; the par $A^{+}$par $B^{+}$plasmid pALA809 construct might produce more ParA than the par $A^{+}$ plasmid pALA824. We have ruled this out as follows: An operon fusion was constructed (pALA811) in a manner similar to that of pALA812 except that this construct expresses ParA in cis because it does not contain the amber mutation in parA. As expected, the expression of this construct shows a modest reduction of expression relative to pALA812 due to the regulatory effect of ParA, and the addition of an extra copy of parA in trans had little effect (Table 4). However, a strong down-regulation was observed when ParB alone was supplied in trans. We conclude that, although ParA alone is capable of exerting a small regulatory effect, the ParB protein plays an important role in the regulation.

\section{The Location of the Regulatory Site}

The above results can be most readily explained if a regulated promoter, upstream of par $A$, were responsible for the transcription of both parA and parB as an operon. A deletion variant of the transcription fusion plasmid pALA812 was isolated that lacks part of the parA open reading frame and material upstream of it that should include the putative parA promoter (pALA815). This deletion reduced lac $Z$ expression some 25 -fold relative to the pALA812 parent. The residual transcription was unregulated by ParA or ParB (Table 4). We conclude that parA and the majority of parB expression is controlled from a promoter located directly upstream of $\operatorname{par} A$ and that the activity of this promoter is regulated by the ParA and ParB proteins. It is likely that this promoter corresponds to the sequence that conforms to the consensus for a strong promoter placed $50 \mathrm{bp}$ upstream of the start of the parA open reading frame (Abeles et al., 1985). It also seems probable that the residual transcription of parB seen in pALA815 comes from a sequence that conforms to the promoter consensus that lies within the carboxy-terminal end of the parA open reading frame (Abeles et al., 1985). Inspection of the data in Tables 3 and 4 suggests that the contribution of this promoter to $p a r B$ expression when the operon is derepressed is negligible, but that when the autoregulatory loop is functioning, its contribution may be more meaningful. Some 20 to $50 \%$ of ParB synthesis appears to originate from the parB promoter when the parA promoter is repressed by the levels of ParA and ParB that are produced by pALA809 (data not shown).

Our results show that, for multicopy plasmids, the equilibrium state of the par operon greatly limits the rate of synthesis of the proteins relative to the maximum synthetic potential. This also appears to be true in the low-copy-number state that is normal for the Pl plasmid. We have found that a single copy of the wild-type par operon, either as an autonomous P1 miniplasmid or as integrated into the host chromosome, is capable of limiting severely the expression of a parA protein fusion despite the fact that the reporter fusion is itself present at high copy number (data not shown). We conclude that the normal equilibrium state of the par operon is severely down-regulated.

\section{DISCUSSION}

We have shown here that the P1 par system encodes two essential proteins. The genes are organized as an operon that is subject to autoregulation. The $E$. coli sex factor $F$ also contains a partition region referred to variously as par (Austin and Abeles, 1983a) or the sop genes (Ogura and Hiraga, 1983). This $F$ region also encodes two proteins (Ogura and Hiraga, 1983) that both appear to be essential for par function (Austin and Wierzbicki, 1983). Moreover, recent studies suggest that the $F$ par genes are also subject to a similar autoregulation (Trawick, unpub- 
lished observations quoted by Kline, 1985). Thus, although the $F$ and $P 1$ par regions show little or no DNA homology, the general organization of the two regions seems to be remarkably similar. Genetic and biochemical studies that compare the properties of these two systems should be important for defining the role of partition proteins in the segregation of plasmids to daughter cells. We are currently attempting to purify the two P1 par proteins to investigate their properties.

We have previously reported that the presence of additional copies of an isolated parA gene within the cell can block par function (Abeles et al., 1985). This is due to the overproduction of the ParA protein, as the introduction of a nonsense mutation in the parA open reading frame of these additional copies abolishes the effect. Thus, ParA appears to be effective for partition only within a fixed range of concentration. Recently, it has been shown that overproduction of the ParB protein is also deleterious to partition (Funnell, 1988). The stringent autoregulation described here for ParA and ParB synthesis may therefore be important for par function. Perhaps these proteins are involved in the morphogenesis of some specific structure that constitutes the equivalent of a mitotic apparatus. The presence of an excess of these proteins relative to each other or other components might then block proper assembly of the structure.

We have shown that both the $A$ and $B$ proteins participate in the repression of par operon transcription. The simplest explanation for this would be that the two proteins, either independently or as a complex, bind to the DNA in the vicinity of the parA promoter and block its activity. Inspection of the DNA sequence in the vicinity of the putative parA promoter reveals the presence of a highly AT-rich $20-$ bp imperfect palindrome that completely spans the region between the -10 promoter region and the likely ribosome binding site for parA (Abeles et al., 1985). We are currently attempting to isolate mutations in this sequence to see if they affect par operon regulation.

\section{ACKNOWLEDGMENTS}

Research sponsored by the National Cancer Institute, DHHS, under Contract No. NO1-CO-74101 with Bionetics Research, Inc. The contents of this publication do not necessarily reflect the views of policies of the Department of Health and Human Services, nor does mention of trade names, commercial products, or organizations imply endorsement by the U.S. Government.

\section{REFERENCES}

AbEles, A. L., SNyder, K. M., AND ChattoraJ, D. K. (1984). Pl plasmid replication: Replicon structure. $J$. Mol. Biol. 173, 307-324.

Abeles, A. L., Friedman, S. A., AND Austin, S. J. (1985). Partition of unit-copy miniplasmids to daughter cells. III. The DNA sequence and functional organization of the $\mathrm{Pl}$ partition region. J. Mol. Biol. 85, 261-272.

AUSTIN, S., AND ABELES, A. (1983a). Partition of unitcopy miniplasmids to daughter cells. I. P1 and F miniplasmids contain discrete, interchangeable sequences sufficient to promote equipartition. $\mathrm{J}$. $\mathrm{Mol}$. Biol. 169, 353-372.

Austin, S., AND ABELES, A. (1983b). Partition of unitcopy miniplasmids to daughter cells. II. The partition region of miniplasmid Pl encodes an essential protein and a centromere-like site at which it acts. $J . \mathrm{Mol}$. Biol. 169, 373-387.

Austin, S., Hart, F., Abeles, A., AND SternberG, N. (1982). Genetic and physical map of a P1 miniplasmid. J. Bacteriol. 152, 63-71.

AUSTIN, S., AND WIERzBICKI, A. (1983). Two mini-Fencoded proteins are essential for equipartition. Plasmid 10, 73-81.

Austin, S. J., Mural, R. J., Chattoraj, D. K., AND ABELES, A. L. (1985). Trans- and cis-acting elements for the replication of $\mathrm{P} 1$ miniplasmids. J. Mol. Biol. 183, 195-202.

Churchward, G., Belin, D., and Nagamine, Y. (1984). A pSC101-derived plasmid which shows no sequence homology to other commonly used cloning vectors. Gene 31, 165-171.

Collins, J., AND PRITChaRD, R. H. (1973). Relationship between chromosome replication and $F^{\prime}$ Lac episome replication in Escherichia coli. J. Mol. Biol. 78, 143-155.

DAS, A., COURT, D., AND ADHYA, S. (1976). Isolation and characterization of conditional lethal mutants of Escherichia coli defective in transcription termination factor rho. Proc. Natl. Acad. Sci. USA 73, 1959-1963.

FUNNELL, B. E. (1988). Mini-P1 plasmid partitioning: Excess ParB protein destabilizes plasmids containing the centromere parS. J. Bacteriol. 170, 954-960.

KELLEY, W. S. (1980). Mapping of the polA locus of Escherichia coli K12: Genetic fine structure of the cistron. Genetics $95,15-38$. 
KLINE, B. C. (1985). A review of mini-F plasmid maintenance. Plasmid 14, 1-16.

KoOP, A. H., Hartley, M. E., and Bourgeols, S. (1987). A low-copy-number vector utilizing $\beta$-galactosidase for the analysis of the gene control elements. Gene 52, 245-256.

MANIATIS, T., FRITSCH, E. F., AND SAMbroOK, J. (1982). "Molecular Cloning: A Laboratory Manual." Cold Spring Harbor Laboratory, Cold Spring Harbor, NY.

Martin, K. A., Friedman, S. A., AND Austin, S. J. (1987). The partition site of the P1 plasmid. Proc. Natl. Acad. Sci. USA 84, 8544-8547.

Miller, J. H. (1972). "Experiments in Molecular Genetics." Cold Spring Harbor Laboratory, Cold Spring Harbor, NY.

OGura, T., and Hiraga, S. (1983). Partition mechanism of $F$ plasmid: Two plasmid gene-encoded prod- ucts and a cis-acting region are involved in partition. Cell 32, 351-360.

PrentKi, P., Chandler, M., and Caro, L. (1977). Replication of the prophage P1 during the cell cycle of Escherichia coli. Mol. Gen. Genet. 152, 71-76.

Silhavy, T. J., Berman, M. L., AND ENQUist, L. W. (1984) "Experiments in Gene Fusion," p. 250. Cold Spring Harbor Laboratory, Cold Spring Harbor, NY.

SOM, T., AND TOMIZAWA, J. (1982). Origin of replication of Escherichia coli plasmid RSF1030. Mol. Gen. Genet. 187, 375-383.

WEINSTOCK, G. M., ap RHYS, C., BERMAN, M. L., HAMPaR, B., JaCkson, D., Silhavy, T. J., WeiseMANN, J., AND ZWEIG, M. (1983). Open reading frame expression vectors: A general method for antigen production in Escherichia coli using protein fusions to $\beta$-galactosidase. Proc. Natl. Acad. Sci. USA 80, 4432-4436.

Communicated by Kurt Nordström 